\title{
Pancreaticogastrostomy: A Safe Option in Pancreaticoduodenectomy for Pancreatic Head and Periampullary Neoplasms
}

\author{
Muhammad Asif Noor, Faisal Hanif, Osama Shakeel and Hassaan Bari \\ Department of Surgical Oncology, Shaukat Khanum Memorial Cancer Hospital and Research Centre, Lahore, Pakistan
}

\begin{abstract}
Objective: To determine the safety of pancreaticogastrostomy in pancreaticoduodenectomy in patients with periampullary and pancreatic head neoplasms in terms of surgical technique, pancreatic fistula rate, 30 days mortality and three years survival.

Study Design: Cohort study.

Place and Duration of Study: Shaukat Khanum Memorial Cancer Hospital and Research Centre, Lahore, Pakistan, from October 2014 to September 2017.

Methodology: Patients undergoing pancreaticoduodenectomy for pancreatic head and periampullary tumors were included. Patients having metastatic disease or involvement of celiac artery, hepatic artery or superior mesenteric artery on preoperative scans, complete encasement of portal vein and superior mesenteric vein (SMV) were excluded. Patients' characteristics including the demographics, surgical technique, postoperative pancreatic fistula, 30 days mortality and three years survival were recorded. Mean \pm standard deviation was used for continuous variables while frequencies and percentages were used for categorical variables. Kaplan-Meier method was used to estimate survival as a function of time, and survival differences were analysed by either Log-Rank test or Tarone-Ware test. Statistical significance was defined as a two-tailed $p$-value 0.05 .

Results: One hundred and one patients underwent pancreaticoduodenectomy. Fifty-eight (57.4\%) were males and $43(42.4 \%)$ were females $(n=43)$. Mean age was $51.5 \pm 14.17$ years. The commonly found tumor was periampullary adenocarcinoma which was present in $49.5 \%(n=50)$ patients followed by pancreatic head adenocarcinoma which was present in $32.7 \%(n=33)$ patients and $17.8 \%(n=18)$ patients had other tumors. Most common pathological T-stage was T3 present in $47.5 \%(n=48)$ patients, followed by T2 found in $36.6 \%(n=37)$ patients and T1 stage was present in $15.8 \%$ $(n=16)$ patients. $57.4 \%(n=58)$ patients had node positive disease. Pancreaticogastrostomy was done in $87.13 \%(n=88)$ patients, while pancreaticojejunostomy was done in $12.87 \%(n=13)$ patients. Recurrent disease was noticed in $11.9 \%$ $(n=12)$ patients. Mean survival of pancreatic head adenocarcinoma was $787.04 \pm 81.89$ days, which was comparatively less than periampullary adenocarcinoma, i.e. $983.10 \pm 52.27$ days $(p=0.08)$. Overall mean survival was $924 \pm 41.3$ days. Conclusion: Patients with periampullary tumors had a better outcome than pancreatic head tumors in this series. Pancreaticogastrostomy can be a safe alternative to pancreaticojejunostomy, especially in patients having non-dilated pancreatic duct and soft pancreas.
\end{abstract}

Key Words: Whipple's procedure, Pancreaticoduodenectomy, Pancreatic tumors, Periampullary tumors, Pancreaticogastrostomy(PG).

How to cite this article: Noor MA, Hanif F, Shakeel O, Bari H. Pancreaticogastrostomy: A safe option in pancreaticoduodenectomy for pancreatic head and periampullary neoplasms. J Coll Physicians Surg Pak 2020; 30(1):51-56.

\section{INTRODUCTION}

Pancreatic cancer is one of the most aggressive malignancies nowadays. ${ }^{1}$ It accounts for $3 \%$ of new cases per annum,,$^{2}$ and it is fourth leading cause of deaths in the west due to malignancy. Pancreatic cancer has a poor prognosis with 5 years survival $<5 \%, 3$ despite active surgical treatment. With newer modalities of treat-ment,

Correspondence to: Dr. Faisal Hanif, Consultant Hepatobiliary and Pancreatic Surgeon, Shaukat Khanum Memorial Cancer Hospital and Research Centre, Johar Town, Lahore, Pakistan E-mail: faisal.hanif@ymail.com

Received: June 19, 2019; Revised: 18 October, 2019; Accepted: 18 October, 2019 outcomes of pancreatic cancer still remain poor and has changed very little in the last three decades. Surgery is the mainstay of treatment; but adjuvant chemotherapy is essential for long term survival. Risk factors for pancreatic cancer are smoking, ${ }^{4}$ alcohol, chronic pancreatitis and diabetes mellitus, ${ }^{5}$ however exact cause remains unknown.

With the advancement of cross-sectional imaging technology, more pancreatic and periampullary tumors are being diagnosed, thus leading to more pancreatic resections.6,7 Pancreaticoduodenectomy is the surgical procedure of choice for benign and malignant periampullary and pancreatic head tumors. ${ }^{8}$ It is a complex surgical procedure associated with major complications including pancreatic fistula, delayed gastric emptying, 
intra-abdominal collection, bleeding and pulmonary complications. ${ }^{9}$ With recent advance-ment in perioperative care, mortality rates after pancreatic resection have been reduced to less than 5\%.10 After surgical resection, the treatment failure is mainly due to metastasis and local recurrence. Postoperative morbidity remains as high as $30-50 \%$ even in high volume centres different techniques are used in these cases. ${ }^{11}$ Postoperative pancreatic fistula (POPF) is the major cause of postoperative morbidity and its incidence is $9 \%$ to $29 \% .12$ POPF significantly affects the patient's quality of life and also increases the treatment cost. Hospital stay is prolonged and postoperative mortality is also increased. ${ }^{13}$ To reduce the incidence of POPF, different techniques were used for pancreatico-enteric anastomosis. Most commonly used techniques are pancreaticogastrostomy (PG) and pancreaticojejunostomy (PJ). Although some studies favour PG over PJ, but there is no consensus about the best technique. ${ }^{14-16}$ The rationale of this study was to share an evidence-based experience of pancreaticoduodenectomy in these cases.

The objective of the study was to determine the safety of pancreaticogastrostomy in pancreaticoduodenectomy in patients with periampullary and pancreatic head neoplasms in terms of surgical technique, pancreatic fistula rate, 30 days mortality and three years survival.

\section{METHODOLOGY}

This study of 101 patients was carried out at Shaukat Khanum Memorial Cancer Hospital and Research Centre (SKMCH \& RC) Lahore, from October 2014 to September 2017. Patient's information like age, gender, site of tumor, disease recurrence, TNM staging, surgical technique, and postoperative complications were recorded. Patients having resectable periampullary or pancreatic head tumors were included. Patients having metastatic disease or involvement of celiac artery, hepatic artery or superior mesenteric artery on preoperative scans, were excluded. Patients with complete encasement of portal vein and superior mesenteric vein (SMV) were also excluded from the study. The ethical approval was sought from Institutional Review Board (IRB) of SKMCH \& RC. Data was collected through human information system (HIS), electronic database of SKMCH \& RC.

In all patients, cytological or histological diagnoses of malignancy were confirmed by means of endoscopic biopsy, biliary brush cytology or endoscopic ultrasound, guided FNAC. Computed tomography (CT) scan and endoscopic ultrasound were performed in all patients for preoperative staging of the disease. All patients were discussed in multidisciplinary team meeting before starting any treatment.

Standard pancreaticoduodenectomy(PD) (Resection of distal stomach, duodenum, pancreatic head, gall bladder and $\mathrm{CBD}$ ) was performed in all patients. For reconstruction pancreaticojejunostomy (PJ) and pancreaticogastrostomy $(P G)$ techniques were used. In PG reconstruction, an opening was made in the posterior wall of stomach and pancreatic stump was invaginated into the stomach. This end-to-side pancreaticogastrostomy was secured with non-absorbable interrupted sutures. For PJ reconstruction, a two layered end-to-side anastomosis was designed with interrupted non-absorbable sutures. A stent in the pancreatic duct was placed when the duct was dilated and could accommodate at least a 5-6 Fr. feeding tube. After PG or PJ reconstruction, end-to-side hepaticojejunostomy was performed followed by gastrojejunostomy and entero-enterostomy. Two abdominal drains were placed in every patient. Nasogastric and nasojejunal tubes were inserted after surgery.

On the first postoperative day, oral sips of water was started and nasogastric tube was removed. Amylase levels of drain fluids were checked on third postoperative day. If amylase levels were found to be normal then oral free fluids were started and progressed to soft diet. When patient's postoperative pain was controlled on oral analgesia and they had started taking solid diet then they were discharged from the hospital.

Postoperative complications, needing surgical or medical treatment, were recorded; especially postoperative pancreatic fistula (POPF). Perioperative mortality was defined as any death, regardless of the cause, occurring in the same admission or within 30 days of surgery. All patients were operated by the same surgeon $(\mathrm{FH})$.

POPF was classified and defined according to International Study Group of Pancreatic Fistula (ISGPF) classification. It was defined as if drain fluid amylase levels were more than three times of upper normal serum value after the third postoperative day. 17 According to ISGPF, POPF was classified into three grades of severity.

Grade A pancreatic fistula (biochemical leak) was considered when there was no peripancreatic collection on imaging and patient was clinically well, but there is delayed removal of abdominal drains. Postoperative management was not changed in these cases.

Grade B pancreatic fistula was considered when patient was doing well clinically, but needed some special measures like placement or repositioning of peripancreatic drains leading to prolonged hospital stay. Patients were discharged with drains and followed up as outpatient.

Grade C pancreatic fistula was labelled when patient was unwell clinically and medical or surgical intervention was required. Major change was required in management plan including prolonged hospital stay, intensive care support and increased chances of mortality.

Initially, adjuvant chemotherapy was given according to ESPAC-3 trial, 18 which was changed after the results of ESPAC-4 trial. 19 
SPSS software (version 20) was used for statistical analysis of the data. Mean \pm standard deviation was determined for continuous variables while frequencies and percentages were determined for categorical variables. The Kaplan-Meier method estimated survival as a function of time; and survival differences were analysed by either Log-Rank test or Tarone-Ware test. Statistical significance was defined as a two-tailed p-value 0.05 .

\section{RESULTS}

In our study, 101 patients underwent pancreatico-duodenectomy in three years. Among them, $57.4 \% \quad(n=58)$ were males and $42.4 \% \quad(n=43)$ were females. The commonly found tumor was periampullary tumor, which was present in $49.5 \% \quad(n=50)$ patients followed by pancreatic head adenocarcinoma, which was present in $32.7 \%(n=33)$ patients; and $17.8 \%(n=18)$ patients had other tumors. Mean age was $51.5 \pm 14.17$ years. The most common pathological T-stage was T3 present in $47.5 \%(n=48)$ patients, followed by T2 found in $36.6 \%$ $(n=37)$ patients, and T1 stage was present in $15.8 \%$

Table I: Patient demographics and tumor characteristics.

\begin{tabular}{|c|c|}
\hline Variable & Frequency $\mathrm{N}(\%)$ \\
\hline Age (mean \pm SD) & $51.5 \pm 14.17$ \\
\hline Up to 40 & $24(23.8 \%)$ \\
\hline Above 40 & $77(76.2 \%)$ \\
\hline \multicolumn{2}{|l|}{ Gender } \\
\hline Female & $43(42.6 \%)$ \\
\hline Male & $58(57.4 \%)$ \\
\hline \multicolumn{2}{|l|}{ Site } \\
\hline Pancreatic tumor & $33(32.7 \%)$ \\
\hline Periampullary tumor & $50(49.5 \%)$ \\
\hline Others & $18(17.8 \%)$ \\
\hline \multicolumn{2}{|l|}{ Recurrence } \\
\hline No & $89(88.1 \%)$ \\
\hline Yes & $12(11.9 \%)$ \\
\hline \multicolumn{2}{|l|}{ Pathological T stage } \\
\hline $\mathrm{T} 1$ & $16(15.8 \%)$ \\
\hline $\mathrm{T} 2$ & $37(36.6 \%)$ \\
\hline $\mathrm{T} 3$ & $48(47.5 \%)$ \\
\hline \multicolumn{2}{|l|}{ Pathological N stage } \\
\hline No & $43(42.6 \%)$ \\
\hline $\mathrm{N} 1$ & $58(57.4 \%)$ \\
\hline \multicolumn{2}{|l|}{ Death } \\
\hline No & $87(86.1 \%)$ \\
\hline Yes & $14(13.9 \%)$ \\
\hline Median survival (overall) days & 1080 \\
\hline Pancreatic tumor & 930 \\
\hline Periampullary tumor & 1080 \\
\hline Mean survival (overall) days & $924.67 \pm 41.30$ \\
\hline Pancreatic tumor & $787.04 \pm 81.89$ \\
\hline Periampullary tumor & $983.10 \pm 52.27$ \\
\hline
\end{tabular}

Table II: Incidence of post-operative pancreatic fistula.

\begin{tabular}{lc}
\hline Grade A & $14(13.9 \%)$ \\
\hline Grade B & $8(7.9 \%)$ \\
\hline Grade C & $5(5.0 \%)$ \\
\hline
\end{tabular}

$(n=16)$ patients. Nodal involvement was seen in $57.4 \%$ $(n=58)$ patients (Table I).

Pancreaticogastrostomy was performed in $87.13 \%(n=88)$ patients, while PJ was performed in $12.87 \%(n=13)$ patients. Recurrence was observed in $11.9 \%(n=12)$ patients, while $88.1 \%(n=89)$ patients had no recurrence. Mean survival of pancreatic tumors was $787.04 \pm 81.89$ days, while the mean survival of periampullary tumors was $983.10 \pm 52.27$ days $(p=0.08)$. Overall mean survival was $924 \pm 41.3$ days (Figure 1A). Disease-free survival of patients with periampullary tumor was better than patients with pancreatic head tumor (Figure 1B). Estimated three years survival of T1 and T2 tumors was about $85 \%$, while T3 tumor had estimated survival of $40 \%$ at three years (Figure 2A). Similarly, T1 and T2 tumors had better disease-free survival than T3 tumors (Figure 2B). Overall estimated survival of node negative disease was about $85 \%$ while node positive disease had about $50 \%$ survival (Figure 2C). Patients with node negative disease had better disease free survival than patients who had node positive disease (Figure 2D).

POPF was noted in 27 patients (Table II). Type A fistula was observed in $14(13.9 \%)$ patients. Eight $(7.9 \%)$ patients developed type $B$ fistula, while type $C$ fistula was seen in $5(5 \%)$ patients. There was one death recorded within 30 days of postoperative period.

\section{DISCUSSION}

Despite advancements in the perioperative care, pancreaticoduodenectomy is associated with high morbidity and mortality that is about $>50 \%$ and $2 \%$, respectively. 20 But with recent advancement in surgical technique, better understanding of disease process and critical care, this procedure is adopted worldwide for the treatment of pancreatic head and periampullary neoplasms.

Large tumor size and old age are no more contraindications of surgery. Even in elderly population, survival benefit is same after resection. The results are comparable with published international literatures in terms of 30 days mortality and morbidity. ${ }^{21}$

A lot of work has been done in the last two decades to reduce frequency of POPF, including different techniques of pancreatic anastomosis like PG, PJ, duct to mucosa and invagination technique. Although, no consensus has been made about the best technique, but some studies have shown that PG is better than PJ.22-24 Apart from surgical technique, texture of pancreas is also a contributing factor in terms of developing POPF. Studies have shown that non-dilated pancreatic duct and soft pancreas are associated with higher chances of POPF. 13,25

Portal vein or SMV involvement by the tumor is no more a contraindication to surgery. Perioperative morbidity and survival after venous resection and reconstruction 


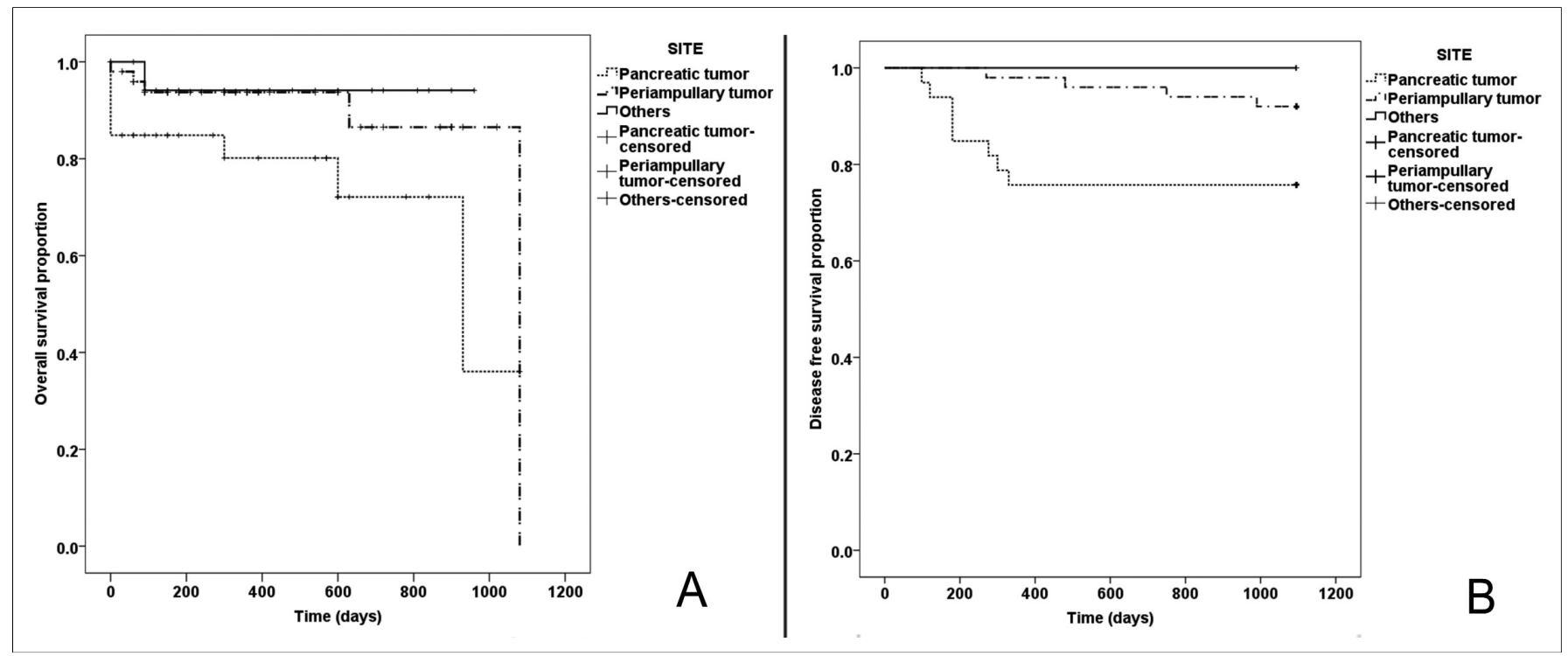

Figure 1: (A) Overall survival with tumor site. (B) Disease free survival with tumor site.

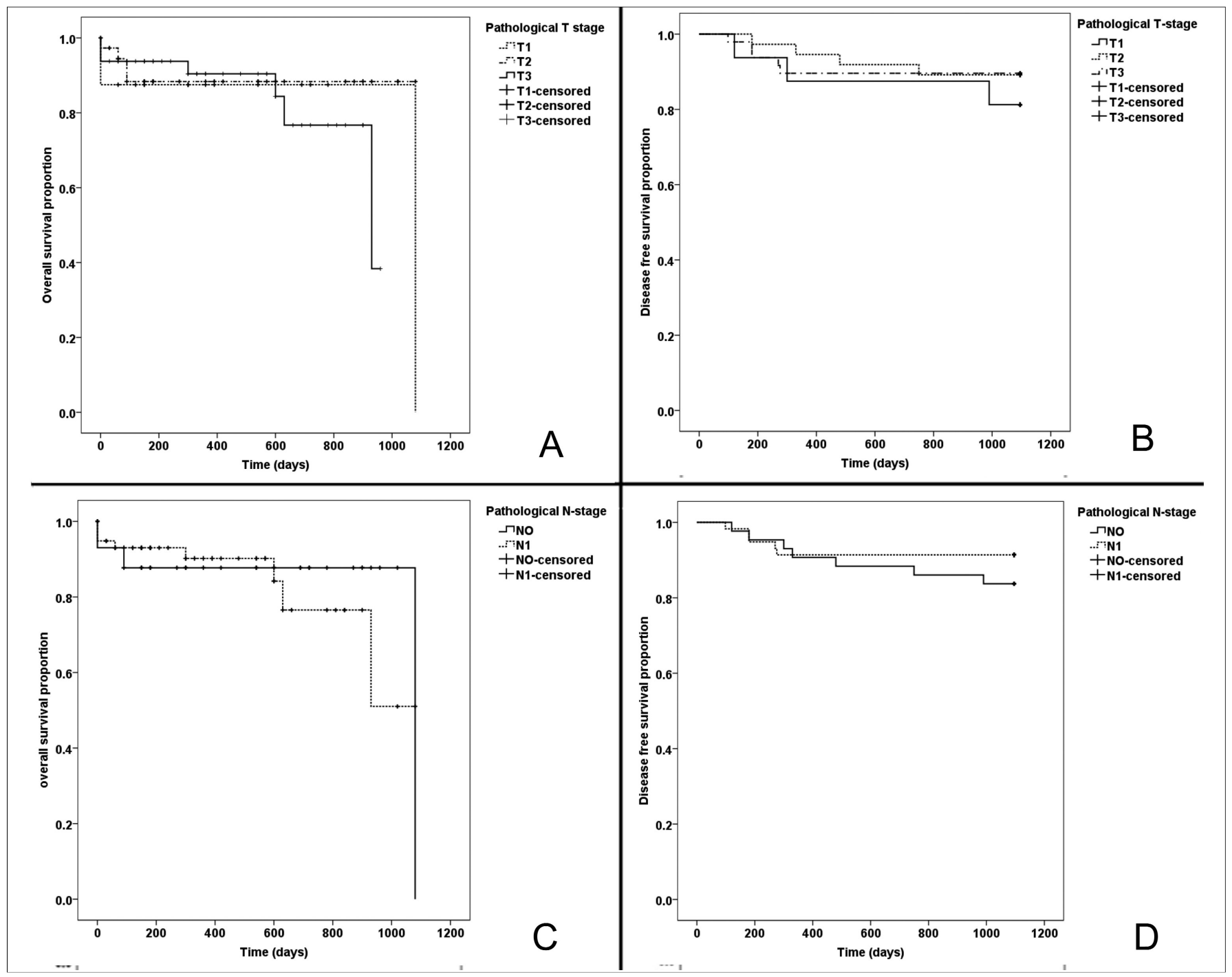

Figure 2: (A) Overall survival with T-stage. (B) Disease-free survival with T-stage. (C) Overall survival with N-stage. (D) Disease-free survival with N-stage. 
have been found to be comparable to standard resection. 25 Vascular anomalies should be identified preoperatively with a tri-phasic CT scan to avoid any inadvertent injury to hepatic arteries.

Achieving a low morbidity and mortality rate after surgery is a function of individual surgeon's experience as well as the volume of institution. High volume centres tend to have more surgeons specialising in specific procedures, more consistent postoperative care, betterstaffed ICUs, and greater resources to deal with postoperative complications.

Patient's selection as well as preoperative evaluation and optimisation, surgical technique and postoperative care are extremely significant not only in reducing patient's mortality and morbidity but also for long term oncological outcome. A dedicated hepato-pancreatobiliaray unit and a reasonable level of experience along with multidisciplinary treatment approach is thus necessary to achieve such good results.

Despite advancement in chemotherapeutic regimens, surgical resection still remains mainstay of treatment for pancreatic head and periampullary neoplasms. This study shows that early tumor stage, absence of metastases to lymph nodes and periampullary tumor site are strong prognostic indicators for patient survival after pancreaticoduodenectomy. Pancreaticogastrostomy was the preferred technique of reconstruction in this study. POPF rate and perioperative mortality is comparable with international published data. Hence, this technique of reconstruction is a safe alternative to pancreaticojejunostomy after pancreaticoduodenectomy.

The new finding in this study is that three years survival of periampullary tumors was much better than pancreatic head tumors. When compared with international literature, periampullary tumor has better survival rate. The survival of pancreatic head tumor is comparable with published data. 20 Currently, there is no prognostic tool to predict survival according to these neoplasms originating from different sites. Further studies can be done focusing on tumor biology and the site of tumor with its impact on patient's overall and disease-free survival. This can also lead to separate perioperative treatment strategies according to specific tumor biology and site of the disease.

This study has its limitations. First of all, this is a retrospective study. There is no comparison of choice of procedure. The follow-up is of short duration. There needs to be a follow-up study, which can focus on 5 years overall and disease-free survival.

\section{CONCLUSION}

Pancreaticoduodenectomy has now been considered a safe procedure and has better outcomes. Pancreaticogastrostomy is a safe alternative to pancreaticojejunostomy in patients with non-dilated pancreatic duct and soft pancreas. Survival of periampullary tumors is better than pancreatic head tumors.

\section{ETHICAL APPROVAL:}

Ethical approval was obtained from Institutional Review Board (IRB) of Shaukat Khanum Memorial Cancer Hospital and Research Centre, prior to the commencement of study.

\section{PATIENTS' CONSENT:}

Informed consent was taken from every patient about data collection, analysis and research publication.

\section{CONFLICT OF INTEREST:}

Authors declared no conflict of interest.

\section{AUTHORS' CONTRIBUTION:}

MAN: Study lead, manuscript writing, data collection and analysis.

FH: Concept, study design and supervision.

OS: Data collection, analysis and review of manuscript. HB: Data collection.

\section{REFERENCES}

1. Wolfgang CL, Herman JM, Laheru DA, Klein AP, Erdek MA, Fishman EK, et al. Recent progress in pancreatic cancer. $C A$ Cancer J Clin 2013; 63:318-48.

2. Jemal A, Siegel R, Xu J, Ward E. Cancer statistics, 2010. CA Cancer J Clin 2010; 60:277-300.

3. Ryan DP, Hong TS, Bardeesy N. Pancreatic adenocarcinoma. N Engl J Med 2014; 371:1039-49.

4. Wang $Y$, Duan H, Yang X, Guo J. Cigarette smoking and the risk of pancreatic cancer: A case-control study. Med Oncol 2014; 31:184.

5. Bilimoria KY, Bentrem DJ, Ko CY, Ritchey J, Stewart AK, Winchester DP, et al. Validation of the $6^{\text {th }}$ edition AJCC pancreatic cancer staging system: Report from the National Cancer Database. Cancer 2007; 110:738-44.

6. Long J, Luo GP, Xiao ZW, Liu ZQ, Guo M, Liu L, et al. Cancer statistics: Current diagnosis and treatment of pancreatic cancer in Shanghai, China. Cancer Lett 2014; 346:273-7.

7. Kaur S, Baine MJ, Jain M, Sasson AR, Batra SK. Early diagnosis of pancreatic cancer: Challenges and new developments. Biomark Med 2012; 6:597-612.

8. Srinarmwong C, Luechakiettisak P, Prasitvilai W. Standard Whipple's operation versus pylorus preserving pancreaticoduodenectomy: A randomized controlled trial study. J Med Assoc Thai 2008; 91:693-8.

9. Karim SA, Abdulla KS, Abdulkarim QH, Rahim FH. The outcomes and complications of pancreaticoduodenectomy (Whipple procedure): Cross sectional study. Int J Surg 2018; 52:383-7.

10. McPhee JT, Hill JS, Whalen GF, Zayaruzny M, Litwin DE, Sullivan ME, et al. Perioperative mortality for pancreatectomy: A national perspective. Ann Surg 2007; 246:246-53.

11. Satoi S, Toyokawa H, Yanagimoto H, Yamamoto T, Yamao J, $\mathrm{Kim} \mathrm{S}$, et al. A new guideline to reduce postoperative morbidity after pancreaticoduodenectomy. Pancreas 2008; 37:128-33. 
12. Binziad S, Salem AA, Amira G, Mourad F, Ibrahim AK, Manim TM. Impact of reconstruction methods and pathological factors on survival after pancreaticoduodenectomy. South Asian J Cancer 2013; 2:160-8.

13. Yardimci S, Kara YB, Tuney D, Attaallah W, Ugurlu MU, Dulundu E, et al. A simple method to evaluate whether pancreas texture can be used to predict pancreatic fistula risk after pancreatoduodenectomy. J Gastrointest Surg 2015; 19:1625-31.

14. Zhu F, Wang M, Wang X, Tian R, Shi C, Xu M, et al. Modified technique of pancreaticogastrostomy for soft pancreas with two continuous hemstitch sutures: A single-center prospective study. J Gastrointest Surg 2013; 17:1306-11.

15. Figueras J, Sabater L, Planellas P, Muñoz-Forner E, LopezBen S, Falgueras $\mathrm{L}$, et al. Randomized clinical trial of pancreaticogastrostomy versus pancreaticojejunostomy on the rate and severity of pancreatic fistula after pancreaticoduodenectomy. Br J Surg 2013; 100:1597-605.

16. He T, Zhao Y, Chen Q, Wang X, Lin H, Han W. Pancreaticojejunostomy versus pancreaticogastrostomy after pancreaticoduodenectomy: A systematic review and meta-analysis. Dig Surg 2013; 30:56-69.

17. Facy $O$, Chalumeau $C$, Poussier $M$, Binquet $C$, Rat $P$, OrtegaDeballon P. Diagnosis of postoperative pancreatic fistula. $\mathrm{Br} \mathrm{J}$ Surg 2012; 99:1072-5.

18. Neoptolemos JP, Moore MJ, Cox TF, Valle JW, Palmer DH, McDonald AC, et al. Effect of adjuvant chemotherapy with fluorouracil plus folinic acid or gemcitabine vs observation on survival in patients with resected periampullary adenocarcinoma: The ESPAC-3 periampullary cancer randomized trial. JAMA 2012; 308:147-56.

19. Neoptolemos JP, Palmer D, Ghaneh P, Valle JW, Cunningham D, Wadsley J, et al. ESPAC-4: A multicenter, international, open- label randomized controlled phase III trial of adjuvant combination chemotherapy of gemcitabine (GEM) and capecitabine (CAP) versus monotherapy gemcitabine in patients with resected pancreatic ductal adenocarcinoma. J Clin Oncol 2016; 34:18.

20. De Pastena M, Paiella S, Marchegiani G, Malleo G, Ciprani D, Gasparini C, et al. Postoperative infections represent a major determinant of outcome after pancreaticoduodenectomy: Results from a high-volume center. Surgery 2017; 162: 792-801.

21. Riall TS, Kuo $Y$, Townsend CM, Freeman J, Nealon W, Goodwin JS. QS48. The impact of age on surgical resection rates and long-term survival in patients with locoregional pancreatic cancer. J Surg Res 2009; 151:284.

22. Hackert T, Büchler MW. Randomized clinical trial of isolated Roux-en-Y versus conventional reconstruction after pancreaticoduodenectomy. Br J Surg 2014; 101:1084-91.

23. Lee JY, Kim EY, Lee JS, Lee SH, Na GH, Hong TH, et al. A novel pancreaticogastrostomy method using only two transpancreatic sutures: Early postoperative surgical results compared with conventional pancreaticojejunostomy. Ann Surg Treat Res 2015; 88:299-305.

24. Shah MF, Pirzada MT, Nasir IU, Malik AA, Farooq U, Anwer AW, et al. Surgical outcome of pancreaticoduodenectomy in pancreatic and periampullary neoplasms. J Coll Physicians Surg Pak 2017; 27:559-62.

25. Elberm H, Ravikumar R, Sabin C, Hilal MA, Al-Hilli A, Aroori S, et al. Outcome after pancreaticoduodenectomy for T3 adenocarcinoma: A multivariable analysis from the UK Vascular Resection for Pancreatic Cancer Study Group. Eur J Surg Oncol 2015; 41:1500-7.

-........... 